Radiologe 2009 · 49:479-480

DOI 10.1007/s00117-009-1887-y

Online publiziert: 19. Juni 2009

๑) Springer Medizin Verlag 2009

\author{
U. Linsenmaier · M. Reiser \\ Institut für Klinische Radiologie, Campus Innenstadt, \\ Klinikum der Ludwig-Maximilians-Universität München
}

\title{
MSCT in der Notfalldiagnostik
}

Das American College of Surgeons hat bereits im Jahr 1913 die Notwendigkeit erkannt, dass es bei der Behandlung von Notfällen zu einer Spezialisierung innerhalb der Medizin kommen müsse und hat in diesem Zusammenhang erste grundlegende Prinzipien der Notfallversorgung publiziert. Folgerichtig wurde 1922 durch Charles L. Scutter in dieser Gesellschaft das erste Committee of Trauma gegründet. Ein wesentlicher Durchbruch in der Notfallbehandlung gelang 1976 durch Dr. R. Adams Cowley, der die Prinzipien der "golden hour" nach einem Unfallereignis entwickelte. Dr. Cowley, ein Begründer der modernen Notfallmedizin, war 1961 auch Initiator des ersten reinen Unfallkrankenhauses in den USA, das an der University of Maryland in Baltimore entstand. Anfang der 199oer Jahre folgte auch in der amerikanischen Röntgengesellschaft RSNA die Gründung einer Fachgesellschaft für Notfallradiologie, die American Society of Emergency Radiology (ASER).

Seit den 199oer Jahren vollzieht sich eine rasante, von zahlreichen Innovationen getriebene technische Entwicklung im Bereich der bildgebenden Notfalldiagnostik. Mit Einführung der Mehrschichtcomputertomographie (MSCT), deren robuste Technik und immer bessere Verfügbarkeit, kam es zu einem Paradigmenwechsel hin zu einer immer früheren und umfassenderen bildgebenden Notfalldiagnostik. Moderne Computertomographen ermöglichen mit 16-64 Detektorzeilen die Untersuchung großer Körpervolumina mit bis zu $200 \mathrm{~cm}$ Scanlänge in weniger als $30 \mathrm{~s}$; erst dadurch wurden beispielsweise bei Patienten mit Polytrauma CT-Ganzkörperuntersuchungen in hoher Qualität möglich. In der Schlaganfalldiagnostik er- laubt die moderne Perfusions-CT inzwischen die lückenlose Abbildung des gesamten Zerebrums. Auch das Herz, die Koronararterien und herznahe Gefäße können im Notfall in höchster räumlicher und zeitlicher Auflösung dargestellt werden. Die jüngste MSCT-Scannergeneration verfügt über bis zu $160 \mathrm{~mm}$ Detektorlänge, 320 Detektorzeilen bei nur noch 0,2-0,3 s Röhrenrotationszeit. Ein Ende dieser imposanten technischen Entwicklung ist noch nicht in Sicht.

Neben der technischen Innovation kam es zu einer Integration der Radiologie in die Notfallmedizin. So sind Radiologen inzwischen anerkannte Partner in den Traumateams in nahezu allen großen Unfallkrankenhäusern und Notfallambulanzen. Gleichzeitig verlässt sich eine ganze Generation jüngerer Notfallmediziner auf die Schnittbilddiagnostik. Mehr als 50\% der Patienten, die über eine Notaufnahme aufgenommen werden, werden mit mindestens einem bildgebenden Verfahren untersucht. Mehrere Untersuchungen haben inzwischen gezeigt, dass die CT-Notfalldiagnostik positiv mit der Vermeidung unnötiger chirurgischer Eingriffe korreliert, einer Vermeidung stationärer Krankenhausaufnahmen und einer Verkürzung des Krankenhausaufenthalts selbst.

Der Einsatz der CT in der Notfalldiagnostik weist jährliche Zuwachsraten von $10-20 \%$ auf und hat sich bereits in den vergangenen 5 Jahren nahezu verdoppelt. Die MSCT hat sich dabei zu einer herausragenden Triagetechnik für akut erkrankte oder verletzte Patienten entwickelt und hat weniger sensitive Verfahren, wie die Projektionsradiographie und die Ultraschalldiagnostik, bereits in einigen Berei- 
chen verdrängt. Die Mehrzahl der Experten geht heute davon aus, dass der Einsatz der MSCT in der Notfalldiagnostik, insbesondere auch außerhalb der regulären Dienstzeiten, noch zunehmen wird. Viele radiologische Abteilungen und Kliniken geraten bereits heute an die Grenzen ihrer Belastbarkeit, denn eine kompetente Durchführung und Befundung dieser Untersuchungen ist unverzichtbar geworden. Im angloamerikanischen Raum besteht seit geraumer Zeit die Forderung, dass eine spezifische radiologische Notfalldiagnostik $24 \mathrm{~h} / 7$ Tage in der Woche auf hohem klinischem und technischem Niveau verfügbar sein muss.

Durch zahlreiche Publikationen und Fortbildungsmaßnahmen ist auch in Europa die Überzeugung gewachsen, dass es in der Notfalldiagnostik einer weiteren fachlichen und wissenschaftlichen Spezialisierung bedarf. Umfassende Monographien zum Thema „Radiologische Notfalldiagnostik“ sind in den letzten Jahren entstanden; u. a. in Radiographics 2008 (Band 28; Monographic Issue, Oktober 2008) und im European Journal of Radiology 2004 (Band 50, April 2004). Die hier vorliegende Ausgabe des „Radiologen“ mit dem Schwerpunktthema „MSCT in der Notfalldiagnostik“ widmet sich in 6 Beiträgen der aktuellen Diagnostik neurologischer (L. Eftimov, B. Ertl-Wagner), thorakaler (J. Wildberger), abdomineller (N. Zorger), urologischer (M. Scherr), vaskulärer (B. Baumert, M. Sadeghi) und traumatologischer Notfälle (M. Körner). Wir danken den Autoren für ihre Mitwirkung am Gelingen dieses Themenhefts.

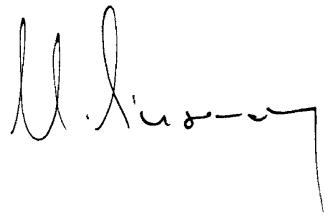

PD Dr. med. Ulrich Linsenmaier

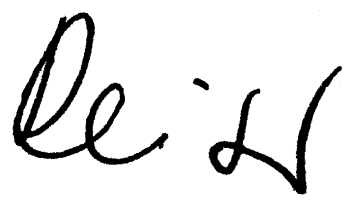

Prof. Dr. Dr. h.c. Maximilian Reiser

Exklusiv für Abonnenten und Gesellschaftsmitglieder :

\section{Nutzen Sie das Online-Archiv von Der Radiologe}

Ihre Vorteile:

- Komfortable und schnelle Recherche nach Themen, Autoren, Suchbegriffen

- Ob unterwegs oder am eigenen PC: Zugriff überall und jederzeit

— Online First: Lesen Sie die aktuellsten Beiträge schon vor Erscheinen des gedruckten Heftes online

\section{Registrieren Sie sich jetzt unter}

www.DerRadiologe.de

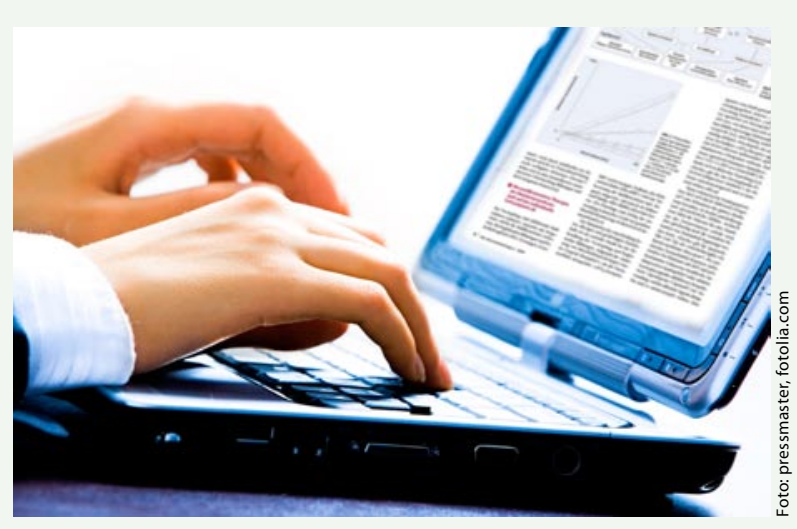

Dieser Online-Service steht auch Mitgliedern der folgenden Fachgesellschaft zur Verfügung:

- Berufsverband der Deutschen Radiologen e.V. 